\title{
POLYNOMIAL VALUES OF (ALTERNATING) POWER SUMS
}

\author{
ANDRÁS BAZSÓ
}

\begin{abstract}
We prove ineffective finiteness results on the integer solutions $x, y$ of the equations

$$
b^{k}+(a+b)^{k}+\cdots+(a(x-1)+b)^{k}=g(y)
$$

and

$b^{k}-(a+b)^{k}+(2 a+b)^{k}-\ldots+(-1)^{x-1}(a(x-1)+b)^{k}=g(y)$,

where $g(x) \in \mathbb{Q}[x], \operatorname{deg} g(x) \geq 3$, and $a \neq 0, b$ are given integers with $\operatorname{gcd}(a, b)=1$.
\end{abstract}

\section{INTRODUCTION AND NEW RESULTS}

Many diophantine problems have been investigated in the literature concerning power sums of consecutive integers. It is well known that the sum

$$
S_{k}(n)=1^{k}+2^{k}+\ldots+(n-1)^{k}
$$

can be expressed by the Bernoulli polynomials $B_{k}(x)$ as

$$
S_{k}(n)=\frac{1}{k+1}\left(B_{k+1}(n)-B_{k+1}\right),
$$

where the polynomials $B_{k}(x)$ are defined by the generating series

$$
\frac{t \exp (t x)}{\exp (t)-1}=\sum_{k=0}^{\infty} B_{k}(x) \frac{t^{k}}{k !}
$$

and $B_{k+1}=B_{k+1}(0)$. Hence $S_{k}$ can be extended to real values $x$, i.e., to the polynomial

$$
S_{k}(x)=\frac{1}{k+1}\left(B_{k+1}(x)-B_{k+1}\right) .
$$

Date: August 11, 2014.

2010 Mathematics Subject Classification. 11D41, $11 \mathrm{~B} 68$.

Key words and phrases. Diophantine equations, Bernoulli polynomials, Euler polynomials. 
A classical problem of Lucas [16], from 1875, was the study of square values of $S_{k}(x)$. Later, in 1956, Schäffer [23] investigated $n$-th power values, that is, the diophantine equation

$$
S_{k}(x)=y^{n} \quad \text { in integers } x, y \text {. }
$$

For $k \geq 1, n \geq 2$ he proved an ineffective finiteness result on the solutions $x, y$ of $(4)$ provided that $(k, n) \notin\{(1,2),(3,2),(3,4),(5,2)\}$. In the exceptional cases $(k, n)$ he proved the existence of infinitely many solutions. Moreover, Schäffer proposed a still unproven conjecture which says that if $(k, n)$ is not in the above exceptional set, then the only nontrivial solution of equation $(4)$ is $(k, n, x, y)=(2,2,24,70)$. In 1980, Györy, Tijdeman and Voorhoeve [13] proved effective finiteness for the solutions of (4) in the general case when, in (4), $n$ is also unknown. Several generalizations of (4) have been considered, e.g. in the papers of Voorhoeve, Györy and Tijdeman [28], Brindza [10], Dilcher [11] and Urbanowicz [25, 26, 27]. Schäffer's conjecture has been confirmed only in a few cases: for $n=2$ and $k \leq 58$ by Jacobson, Pintér and Walsh [15]; and for $n \geq 2$ and $k \leq 11$ by Bennett, Györy and Pintér [5]. For further generalizations of (4) and related results see the survey paper of Györy and Pintér [12] and the references given there.

In [8], Bilu et al. considered the diophantine equations

$$
S_{k}(x)=S_{\ell}(y)
$$

and

$$
S_{k}(x)=y(y+1)(y+2) \ldots(y+(\ell-1)) .
$$

They proved ineffective finiteness results on the solutions $x, y$ of these equations for $k<l$, moreover, they established effective statements for certain small values of $k$ and $l$.

For a positive integer $n \geq 2$ and for $a \neq 0, b$ coprime integers, let

$$
S_{a, b}^{k}(n)=b^{k}+(a+b)^{k}+(2 a+b)^{k}+\ldots+(a(n-1)+b)^{k} .
$$

It is easy to see that the above power sum is related to the Bernoulli polynomials $B_{k}(x)$ in the following way

$$
\begin{aligned}
S_{a, b}^{k}(n)=\frac{a^{k}}{k+1}\left(\left[B_{k+1}\left(n+\frac{b}{a}\right)-\right.\right. & \left.B_{k+1}\right] \\
& \left.-\left[B_{k+1}\left(\frac{b}{a}\right)-B_{k+1}\right]\right) .
\end{aligned}
$$

Thus we can extend $S_{a, b}^{k}$ for every real value $x$ as

$$
S_{a, b}^{k}(x)=\frac{a^{k}}{k+1}\left(B_{k+1}\left(x+\frac{b}{a}\right)-B_{k+1}\left(\frac{b}{a}\right)\right) .
$$


In [14], using a different approach, Howard also obtained relation (9) via generating functions. In the same paper [14], he showed that the alternating power sum

$$
T_{a, b}^{k}(n)=b^{k}-(a+b)^{k}+(2 a+b)^{k}-\ldots+(-1)^{n-1}(a(n-1)+b)^{k}
$$

can be expressed by means of Euler polynomials $E_{k}(x)$ as

$$
T_{a, b}^{k}(n)=\frac{a^{k}}{2}\left(E_{k}\left(\frac{b}{a}\right)+(-1)^{n-1} E_{k}\left(n+\frac{b}{a}\right)\right),
$$

where the classical Euler polynomials $E_{k}(x)$ are defined by the generating function

$$
\frac{2 \exp (x t)}{\exp (t)+1}=\sum_{n=0}^{\infty} E_{n}(x) \frac{t^{n}}{n !} \quad(|t|<\pi) .
$$

For the properties of Bernoulli and Euler polynomials which will be often used in this paper, sometimes without special reference, we refer to the paper of Brillhart [9]. Thus, depending on the power of -1 in (11), we can extend $T_{a, b}^{k}(n)$ to a polynomial in the following two ways:

$$
\begin{aligned}
& \mathrm{T}_{a, b}^{k+}(x)=\frac{a^{k}}{2}\left(E_{k}\left(\frac{b}{a}\right)+E_{k}\left(x+\frac{b}{a}\right)\right), \\
& \mathrm{T}_{a, b}^{k-}(x)=\frac{a^{k}}{2}\left(E_{k}\left(\frac{b}{a}\right)-E_{k}\left(x+\frac{b}{a}\right)\right) .
\end{aligned}
$$

Recently, Bazsó, Kreso, Luca and Pintér [2] generalized the results of Bilu et al. [8] on equation (5) to the equation

$$
S_{a, b}^{k}(x)=S_{c, d}^{\ell}(y)
$$

where $x, y$ are unknown integers, and $k, \ell, a, b, c, d$ are given integers with $0<k<l, \operatorname{gcd}(a, b)=\operatorname{gcd}(c, d)=1$.

In the present paper we study the Diophantine equations

$$
\begin{gathered}
S_{a, b}^{k}(x)=g(y), \\
\mathrm{T}_{a, b}^{k+}(x)=g(y)
\end{gathered}
$$

and

$$
\mathrm{T}_{a, b}^{k-}(x)=g(y),
$$

where $g(y)$ is a rational polynomial of degree at least 3 . These equations have only been investigated in the literature in the case $(a, b)=(1,0)$. Rakaczki [20] and independently Kulkarni and Sury [19] characterized those pairs $(k, g(y))$ for which equation (13) has infinitely many integer solutions. Recently, Kreso and Rakaczki [22] proved an analogous result for equations (14) and (15). For further related results we refer to the papers of Kulkarny and Sury [17, 18], and of Bennett [4]. 
Our goal in the sequel is to extend the results of $[20,19,22]$ to the general case, i.e, to equations (13)-(15). To do this it will be useful to survey what is known about the decomposition properties of the polynomials involved in the equations under consideration.

By a decomposition of a polynomial $F(x)$ over a field $\mathbb{K}$ we mean an equality of the following form

$$
F(x)=G_{1}\left(G_{2}(x)\right) \quad\left(G_{1}(x), G_{2}(x) \in \mathbb{K}[x]\right),
$$

which is nontrivial if

$$
\operatorname{deg} G_{1}(x)>1 \quad \text { and } \quad \operatorname{deg} G_{2}(x)>1 .
$$

Two decompositions $F(x)=G_{1}\left(G_{2}(x)\right)$ and $F(x)=H_{1}\left(H_{2}(x)\right)$ are said to be equivalent if there exists a linear polynomial $\ell(x) \in \mathbb{K}[x]$ such that $G_{1}(x)=H_{1}(\ell(x))$ and $H_{2}(x)=\ell\left(G_{2}(x)\right)$. The polynomial $F(x)$ is called decomposable over $\mathbb{K}$ if it has at least one nontrivial decomposition over $\mathbb{K}$; otherwise it is said to be indecomposable. A detailed discussion on the theory of polynomial decomposition can be found in the monograph of Schinzel [24].

In a recent paper, Bazsó, Pintér and Srivastava [3] proved the following result about the decomposition of the polynomial $S_{a, b}^{k}(x)$ defined above.

Proposition 1. The polynomial $S_{a, b}^{k}(x)$ is indecomposable over $\mathbb{C}$ for even $k$. If $k=2 v-1$ is odd, then any nontrivial decomposition of $S_{a, b}^{k}(x)$ over $\mathbb{C}$ is equivalent to the following decomposition:

$$
S_{a, b}^{k}(x)=\widehat{S}_{a, b}^{v}\left(\left(x+\frac{b}{a}-\frac{1}{2}\right)^{2}\right),
$$

where $\widehat{S}_{a, b}^{v}(x)$ is a rational polynomial of degree $v$.

Proof. This is Theorem 2 of [3].

On equation (13), we prove the following.

Theorem 1. Let $a \neq 0, b \in \mathbb{Z}, \operatorname{gcd}(a, b)=1$ and let $g(x) \in \mathbb{Q}[x]$, $\operatorname{deg} g(x) \geq 3$. Further, let $\alpha, \beta, c \in \mathbb{Q} \backslash\{0\}, \delta(x), q(x) \in \mathbb{Q}[x]$ with $\operatorname{deg} \delta(x)=1, q(x) \neq 0$. Then, for $k>3$ equation (13) has only finitely many integer solutions $x, y$, unless one of the following holds:

(I) $g(x)=S_{a, b}^{k}(q(x))$

(II) $k$ is odd and $g(x)=\widehat{S}_{a, b}^{(k+1) / 2}\left(\delta(x) q(x)^{2}\right)$

(III) $k$ is odd and $g(x)=\widehat{S}_{a, b}^{(k+1) / 2}\left(c \delta(x)^{t}\right)$, where $t \geq 3$ is an odd integer

(IV) $k$ is odd and $g(x)=\widehat{S}_{a, b}^{(k+1) / 2}\left(\left(\alpha \delta(x)^{2}+\beta\right) q(x)^{2}\right)$ 
$(\mathrm{V}) k$ is odd and $g(x)=\widehat{S}_{a, b}^{(k+1) / 2}\left(q(x)^{2}\right)$

with $\widehat{S}_{a, b}^{v}(x)$ specified in Proposition 1.

Our method of proof is based upon Proposition 1 and the general ineffective finiteness criterion of Bilu and Tichy [7] (cf. Proposition 3). Therefore our result is also ineffective.

We note that in the exceptional cases $(I)-(V)$ one can find an equation of the shape (13) having infinitely many integer solutions (see [20] for examples). We further note that for $a=1, b=0, k>3$, our Theorem 1 gives the result of Rakaczki [20, Theorem 1].

The decomposition properties of the polynomials $\mathrm{T}_{a, b}^{k+}(x)$ and $\mathrm{T}_{a, b}^{k-}(x)$ have recently been described in [1] by the present author who proved the following.

Proposition 2. The polynomials $T_{a, b}^{k+}(x)$ and $T_{a, b}^{k-}(x)$ are both indecomposable for any odd $k$. If $k=2 m$ is even, then any nontrivial decomposition of $T_{a, b}^{k+}(x)$ or $T_{a, b}^{k-}(x)$ is equivalent to

$$
\begin{aligned}
T_{a, b}^{k+}(x)=\widehat{T}_{a, b}^{m+}\left(\left(x+\frac{b}{a}-\frac{1}{2}\right)^{2}\right) & \text { or } \\
T_{a, b}^{k-}(x) & =\widehat{T}_{a, b}^{m-}\left(\left(x+\frac{b}{a}-\frac{1}{2}\right)^{2}\right),
\end{aligned}
$$

respectively, where

with

$$
\begin{aligned}
& \widehat{T}_{a, b}^{m+}(x)=\frac{a^{2 m}}{2}\left(E_{2 m}\left(\frac{b}{a}\right)+\tilde{E}_{m}(x)\right), \\
& \widehat{T}_{a, b}^{m-}(x)=\frac{a^{2 m}}{2}\left(E_{2 m}\left(\frac{b}{a}\right)-\tilde{E}_{m}(x)\right)
\end{aligned}
$$

$$
\tilde{E}_{m}(x)=\sum_{n=0}^{m}\left(\begin{array}{c}
2 m \\
2 n
\end{array}\right) \frac{E_{2 n}}{2^{2 n}} x^{m-n} \quad \text { and } \quad E_{j}=2^{j} E_{j}(1 / 2) .
$$

Proof. See [1].

Using Proposition 2 and the finiteness criterion from [7] we prove the following two results on equations (14) and (15), which are again ineffective.

Theorem 2. Let $a \neq 0, b \in \mathbb{Z}, \operatorname{gcd}(a, b)=1$ and let $g(x) \in \mathbb{Q}[x]$, $\operatorname{deg} g(x) \geq 3$. Further, let $\alpha, \beta, c \in \mathbb{Q} \backslash\{0\}, \delta(x), q(x) \in \mathbb{Q}[x]$ with $\operatorname{deg} \delta(x)=1, q(x) \neq 0$. Then, for $k \geq 7$ equation (14) has only finitely many integer solutions $x, y$, unless one of the following holds: 
(I) $g(x)=T_{a, b}^{k+}(q(x))$

(II) $k$ is even and $g(x)=\widehat{T}_{a, b}^{k / 2+}\left(q(x)^{2}\right)$

(III) $k$ is even and $g(x)=\widehat{T}_{a, b}^{k / 2+}\left(\delta(x) q(x)^{2}\right)$

(IV) $k$ is even and $g(x)=\widehat{T}_{a, b}^{k / 2+}\left(c \delta(x)^{t}\right)$, where $t \geq 3$ is an odd integer

$(\mathrm{V}) k$ is even and $g(x)=\widehat{T}_{a, b}^{k / 2+}\left(\left(\alpha \delta(x)^{2}+\beta\right) q(x)^{2}\right)$

with $\widehat{T}_{a, b}^{m+}(x)$ specified in Proposition 2.

Theorem 3. Let $a \neq 0, b \in \mathbb{Z}, \operatorname{gcd}(a, b)=1$ and let $g(x) \in \mathbb{Q}[x]$, $\operatorname{deg} g(x) \geq 3$. Further, let $\alpha, \beta, c \in \mathbb{Q} \backslash\{0\}, \delta(x), q(x) \in \mathbb{Q}[x]$ with $\operatorname{deg} \delta(x)=1, q(x) \neq 0$. Then, for $k \geq 7$ equation (15) has only finitely many integer solutions $x, y$, unless one of the following holds:

(I) $g(x)=T_{a, b}^{k-}(q(x))$

(II) $k$ is even and $g(x)=\widehat{T}_{a, b}^{k / 2-}\left(q(x)^{2}\right)$

(III) $k$ is even and $g(x)=\widehat{T}_{a, b}^{k / 2-}\left(\delta(x) q(x)^{2}\right)$

(IV) $k$ is even and $g(x)=\widehat{T}_{a, b}^{k / 2-}\left(c \delta(x)^{t}\right)$, where $t \geq 3$ is an odd integer

$(\mathrm{V}) k$ is even and $g(x)=\widehat{T}_{a, b}^{k / 2-}\left(\left(\alpha \delta(x)^{2}+\beta\right) q(x)^{2}\right)$

with $\widehat{T}_{a, b}^{m-}(x)$ specified in Proposition 2.

The above two theorems extend Theorem 2 of [22] when $\operatorname{deg} g(x) \geq 3$. We further note that in the exceptional cases $(I)-(V)$ of Theorems 2 and 3 a choice of parameters can be found for which equations (14) resp. (15) have infinitely many integer solutions $x, y$. Such parametric solutions are given in [22] for $a=1, b=0$.

\section{Proof of Theorem 1}

To prove Theorem 1, we need some auxiliary results. First we recall the general ineffective finiteness criterion of Bilu and Tichy [7]. We first define the five kinds of so-called standard pairs of polynomials.

Let $\alpha, \beta$ be nonzero rational numbers, $\mu, \nu, q>0$ and $r \geq 0$ be integers, and let $v(x) \in \mathbb{Q}[x]$ be a nonzero polynomial (which may be constant). Denote by $D_{\mu}(x, \delta)$ the $\mu$-th Dickson polynomial, defined by the functional equation $D_{\mu}(z+\delta / z, \delta)=z^{\mu}+(\delta / z)^{\mu}$ or by the explicit formula

$$
D_{\mu}(x, \delta)=\sum_{i=0}^{\lfloor\mu / 2\rfloor} d_{\mu, i} x^{\mu-2 i} \quad \text { with } \quad d_{\mu, i}=\frac{\mu}{\mu-i}\left(\begin{array}{c}
\mu-i \\
i
\end{array}\right)(-\delta)^{i} .
$$


Two polynomials $f_{1}(x)$ and $g_{1}(x)$ are said to form a standard pair over $\mathbb{Q}$ if one of the ordered pairs $\left(f_{1}(x), g_{1}(x)\right)$ or $\left(g_{1}(x), f_{1}(x)\right)$ belongs to the list below. The five kinds of standard pairs are then listed in the following table.

\begin{tabular}{|l|l|l|}
\hline kind & explicit form of $\left\{f_{1}(x), g_{1}(x)\right\}$ & parameter restrictions \\
\hline first & $\left(x^{q}, \alpha x^{r} v(x)^{q}\right)$ & $\begin{array}{l}0 \leq r<q,(r, q)=1, \\
r+\operatorname{deg} v(x)>0\end{array}$ \\
\hline second & $\left(x^{2},\left(\alpha x^{2}+\beta\right) v(x)^{2}\right)$ & - \\
\hline third & $\left(D_{\mu}\left(x, \alpha^{\nu}\right), D_{\nu}\left(x, \alpha^{\mu}\right)\right)$ & $(\mu, \nu)=1$ \\
\hline fourth & $\left(\alpha^{\frac{-\mu}{2}} D_{\mu}(x, \alpha),-\beta^{\frac{-\nu}{2}} D_{\nu}(x, \beta)\right)$ & $(\mu, \nu)=2$ \\
\hline fifth & $\left(\left(\alpha x^{2}-1\right)^{3}, 3 x^{4}-4 x^{3}\right)$ & - \\
\hline
\end{tabular}

Now we state a special case of the main result of [7], which will be crucial in the proofs of ours.

Proposition 3. Let $f(x), g(x) \in \mathbb{Q}[x]$ be nonconstant polynomials such that the equation $f(x)=g(y)$ has infinitely many solutions in rational integers $x, y$. Then $f=\varphi \circ f_{1} \circ \lambda$ and $g=\varphi \circ g_{1} \circ \mu$, where $\lambda(x), \mu(x) \in \mathbb{Q}[x]$ are linear polynomials, $\varphi(x) \in \mathbb{Q}[x]$, and $\left(f_{1}(x), g_{1}(x)\right)$ is a standard pair over $\mathbb{Q}$.

We recall the following result concerning Bernoulli polynomials $B_{k}(x)$ which is due to Brillhart [9].

Lemma 4. If $k$ is odd, then $B_{k}(x)$ has no multiple roots. For even $k$, the only polynomial which can be a multiple factor of $B_{k}(x)$ over $\mathbb{Q}$ is $x^{2}-x-B$, where $B$ is an odd, positive integer.

The next two lemmas were proved in $[2]$. Let $c_{1}, e_{1} \in \mathbb{Q} \backslash\{0\}$ and $c_{0}, e_{0} \in \mathbb{Q}$.

Lemma 5. The polynomial $S_{a, b}^{k}\left(c_{1} x+c_{0}\right)$ is not of the form $e_{1} x^{q}+e_{0}$ with $q \geq 3$.

Lemma 6. The polynomial $S_{a, b}^{k}\left(c_{1} x+c_{0}\right)$ is not of the form

$$
e_{1} D_{\nu}(x, \delta)+e_{0}
$$

where $D_{\nu}(x, \delta)$ is the $\nu$-th Dickson polynomial with $\nu>4, \delta \in \mathbb{Q} \backslash\{0\}$.

For $P(x) \in \mathbb{C}[x]$, a complex number $c$ is said to be an extremum if $P(x)-c$ has multiple roots. The type of $c$ is defined to be the tuple $\left(\alpha_{1}, \ldots, \alpha_{s}\right)$ of the multiplicities of the distinct roots of $P(x)-c$ in an increasing order. Obviously, $s<\operatorname{deg} P(x)$ and $\alpha_{1}+\ldots+\alpha_{s}=\operatorname{deg} P(x)$.

Proposition 7. For $a \neq 0$ and $k \geq 3, D_{\mu}(x, \alpha)$ has exactly two extrema $\pm 2 \alpha^{\frac{\mu}{2}}$. If $\mu$ is odd, then both are of type $(1,2,2, \ldots, 2)$. If $\mu$ is even, then $2 \alpha^{\frac{\mu}{2}}$ is of type $(1,1,2, \ldots, 2)$ and $-2 \alpha^{\frac{\mu}{2}}$ is of type $(2,2, \ldots, 2)$. 
Proof. See, for instance [6, Proposition 3.3].

Now we are equipped to prove Theorem 1.

Proof of Theorem 1. Let $g(x)$ be a polynomial with rational coefficients and with $\operatorname{deg} g(x) \geq 3$. Suppose that equation (13) has infinitely many solutions in integers $x, y$. Then by Proposition 3 , it follows that there exist $\lambda(x), \mu(x), \varphi(x) \in \mathbb{Q}[x]$ such that

$$
S_{a, b}^{k}(x)=\varphi\left(f_{1}(\lambda(x))\right) \quad \text { and } \quad g(x)=\varphi\left(g_{1}(\mu(x))\right),
$$

where $\left(f_{1}(x), g_{1}(x)\right)$ is a standard pair over $\mathbb{Q}$. Proposition 1 implies that

$$
\operatorname{deg} \varphi(x) \in\left\{1, \frac{k+1}{2}, k+1\right\} .
$$

First, suppose that $\operatorname{deg} \varphi(x)=k+1$. Then, by (18), we observe that $\operatorname{deg} f_{1}(x)=1$. Thus $S_{a, b}^{k}(x)=\varphi(t(x))$, where $t(x) \in \mathbb{Q}[x]$ is a linear polynomial. Clearly, $t^{-1}(x) \in \mathbb{Q}[x]$ is also linear. By (18), we obtain $S_{a, b}^{k}\left(t^{-1}(x)\right)=\varphi\left(t\left(t^{-1}(x)\right)\right)=\varphi(x)$. Hence

$$
g(x)=\varphi\left(g_{1}(\mu(x))\right)=S_{a, b}^{k}\left(t^{-1}\left(g_{1}(\mu(x))\right)\right)=S_{a, b}^{k}(q(x)),
$$

where $q(x)=t^{-1}\left(g_{1}(\mu(x))\right)$. So, if, in our case, equation (13) has infinitely many solutions, then $g(x)$ is of the form as in Theorem 1 (I).

Next we assume that $\operatorname{deg} \varphi(x)=1$. Then there exist $\varphi_{1}, \varphi_{0} \in \mathbb{Q}$ with $\varphi_{1} \neq 0$ such that $\varphi(x)=\varphi_{1} x+\varphi_{0}$. We study now the five kinds of standard pairs. In view of our assumptions on $k$ and $\operatorname{deg} g(x)$, it follows that the standard pair $\left(f_{1}(x), g_{1}(x)\right)$ cannot be of the second kind.

If it is of the third or fourth kind, we then have $S_{a, b}^{k}\left(\lambda^{-1}(x)\right)=$ $e_{1} D_{\mu}(x, \delta)+e_{0}$ for some $e_{0} \in \mathbb{Q}, e_{1}, \delta \in \mathbb{Q} \backslash\{0\}$, which contradicts Lemma 6 since $k=\mu-1>3$.

Now consider the case when, in (18), $\left(f_{1}(x), g_{1}(x)\right)$ is a standard pair of the first kind over $\mathbb{Q}$. Then we have either

(i) $S_{a, b}^{k}\left(\lambda^{-1}(x)\right)=\varphi_{1} x^{t}+\varphi_{0}$, or

(ii) $S_{a, b}^{k}\left(\lambda^{-1}(x)\right)=\varphi_{1} \alpha x^{r} q(x)^{t}+\varphi_{0}$, where $0 \leq r<t,(r, t)=1$ and $r+\operatorname{deg} q(x)>0$.

In the first case (i), we get a contradiction by Lemma 5 since $t=$ $k+1 \geq 5$.

In the second case (ii), we have $g\left(\mu^{-1}(x)\right)=\varphi_{1} x^{t}+\varphi_{0}$. Suppose that $t=\operatorname{deg} g(x)>3$. Then the polynomial $S_{a, b}^{k}\left(\lambda^{-1}(x)\right)-\varphi_{0}$ has a root with multiplicity at least 4 (since $q(x)^{t}$ divides it), which is impossible by Lemma 4 unless $q(x)$ is a constant polynomial. We obtain $r \leq 3$ and $q(x) \equiv Q \in \mathbb{Q} \backslash\{0\}$. It follows that

$$
S_{a, b}^{k}\left(\lambda^{-1}(x)\right)=\varphi x^{r}+\varphi_{0} \text { with } \varphi:=\varphi_{1} \alpha Q^{t} \in \mathbb{Q} \backslash\{0\} .
$$


Lemma 5 implies that $r=k+1=2$ which contradicts $k>3$.

If, in (ii), $t=3$, then we have

$$
S_{a, b}^{k}(x)=\varphi_{1} \alpha \lambda(x)^{r} q(\lambda(x))^{3}+\varphi_{0},
$$

where $r \in\{1,2\}$. If $\operatorname{deg} q(x)=0$, we get back to (20). We can thus assume that $q(x)$ is nonconstant. Using (9), from (21), we derive that

$$
\begin{aligned}
& a^{k} B_{k}\left(x+\frac{b}{a}\right)=\frac{d}{d x} S_{a, b}^{k}(x)= \\
& \quad=\varphi_{1} \alpha \lambda(x)^{r-1} \lambda^{\prime}(x) q(\lambda(x))^{2}\left(r q(\lambda(x))+3 \lambda(x) q^{\prime}(\lambda(x))\right),
\end{aligned}
$$

whence we infer that $q(\lambda(x-b / a))$ is a multiple factor of $B_{k}(x)$ over $\mathbb{Q}[x]$. Then, by Lemma $4, k$ is even and $q(\lambda(x-b / a))=x^{2}-x-B$ for an odd, positive integer $B$. We obtain from (21), that $k=6$ and $r=1$. But in this case $S_{a, b}^{6}(x)-\varphi_{0}$ has a root of multiplicity 3 , thus, by (22), the sixth Bernoulli polynomial $B_{6}(x)$ has a double root. However it is impossible since the discriminant of $B_{6}(x)$ is nonzero.

Finally, suppose that $\left(f_{1}(x), g_{1}(x)\right)$ is a standard pair of the fifth kind. Now (18) implies either

(a) $S_{a, b}^{k}\left(\lambda^{-1}(x)\right)=\varphi_{1}\left(\alpha x^{2}-1\right)^{3}+\varphi_{0}$, or

(b) $S_{a, b}^{k}\left(\lambda^{-1}(x)\right)=\varphi_{1}\left(3 x^{4}-4 x^{3}\right)+\varphi_{0}$.

The second case is impossible, since then we get $k=3$ contradicting our assumption $k>3$.

In the first case (a) we infer that $k=5$ and that $S_{a, b}^{5}\left(\lambda^{-1}(x)\right)-\varphi_{0}$ has a root with multiplicity at least 3 . But the number of roots as well as their multiplicities of a polynomial remain unchanged if we replace the variable $x$ by a linear polynomial of it. Hence we obtain that $S_{a, b}^{5}(x)-\varphi_{0}$ also has a root with multiplicity at least 3 . But then, by

$$
\frac{d}{d x}\left(S_{a, b}^{5}(x)-\varphi_{0}\right)=\frac{d}{d x} \frac{a^{5} B_{6}\left(x+\frac{b}{a}\right)}{6}=a^{5} B_{5}\left(x+\frac{b}{a}\right),
$$

the fifth Bernoulli polynomial $B_{5}(x)$ would have a multiple root, which is a contradiction by Lemma 4 .

Let us consider the remaining case $\operatorname{deg} \varphi(x)=(k+1) / 2$. Clearly, $k$ is then odd, and from (18) we know that $\operatorname{deg} f_{1}(x)=2$. Hence it follows that, in (18), $\left(f_{1}(x), g_{1}(x)\right)$ cannot be a standart pair of the fifth kind. Further, we obtain a nontrivial decomposition of $S_{a, b}^{k}(x)$, which by Proposition 1 implies that there exists a linear polynomial $\ell(x)=\ell_{1} x+\ell_{0}$ over $\mathbb{Q}$ such that

$$
\varphi(x)=\widehat{S}_{a, b}^{(k+1) / 2}(\ell(x)) \quad \text { and } \quad \ell\left(f_{1}(\lambda(x))\right)=\left(x+\frac{b}{a}-\frac{1}{2}\right)^{2} .
$$


Again, we study the unexcluded kinds of standard pairs over $\mathbb{Q}$.

First, we assume $\left(f_{1}(x), g_{1}(x)\right)$ to be a standard pair of the first kind. If $\left(f_{1}(x), g_{1}(x)\right)=\left(x^{t}, \alpha x^{r} p(x)^{t}\right)$ with $r<t,(r, t)=1$ and $r+\operatorname{deg} p(x)>$ 0 , then by $\operatorname{deg} f_{1}(x)=2$, the corresponding standard pair is of the form $\left(f_{1}(x), g_{1}(x)\right)=\left(x^{2}, \alpha x p(x)^{2}\right)$. If $\lambda(x)=\lambda_{1} x+\lambda_{0}$, then (24) takes the form $\ell\left(\left(\lambda_{1} x+\lambda_{0}\right)^{2}\right)=(x+b / a-1 / 2)^{2}$, whence one can deduce that $\ell(x)=x / \lambda_{1}^{2}$. Substituting this to (18), we obtain

$$
g(x)=\widehat{S}_{a, b}^{(k+1) / 2}\left(\ell\left(g_{1}(\mu(x))\right)\right)=\widehat{S}_{a, b}^{(k+1) / 2}\left(\frac{\alpha \mu(x) p(\mu(x))^{2}}{\lambda_{1}^{2}}\right)
$$

So $g(x)$ is of the form as in Theorem 1 (II) with $\delta(x)=\alpha \mu(x) / \lambda_{1}^{2}$ and $q(x)=p(\mu(x))$.

In the switched case $\left(f_{1}(x), g_{1}(x)\right)=\left(\alpha x^{r} p(x)^{t}, x^{t}\right)$, where $r<t$, $(r, t)=1$ and $r+\operatorname{deg} p(x)>0$, we obtain from $\operatorname{deg} f_{1}(x)=2$ that one of the following cases occurs:

(A) $r=0, t=1$ and $\operatorname{deg} p(x)=2$, or

(B) $r=2, t>2$ is odd and $p(x)$ is constant.

In case $(\mathrm{A})$ we have $g_{1}(x)=x$ which together with (18) and (24) implie

$$
\begin{aligned}
& g(x)=\widehat{S}_{a, b}^{(k+1) / 2}\left(\ell\left(g_{1}(\mu(x))\right)\right)= \\
& \quad=\widehat{S}_{a, b}^{(k+1) / 2}(\ell(\mu(x)))=\widehat{S}_{a, b}^{(k+1) / 2}\left(\delta(x) q(x)^{2}\right),
\end{aligned}
$$

where $\delta(x)=\ell(\mu(x))$ and $q(x) \equiv 1$. Thus $g(x)$ is again of the form as in Theorem 1 (II).

If case (B) holds, then we can write $f_{1}(x)=\beta x^{2}$, with $\beta=\alpha p(x)^{t} \in$ $\mathbb{Q} \backslash\{0\}$. Substituting this to $(24)$, we deduce that $\ell(x)=x /\left(\beta \lambda_{1}^{2}\right)$, whence, by (18), we get

$$
\begin{aligned}
& g(x)=\widehat{S}_{a, b}^{(k+1) / 2}\left(\ell\left(g_{1}(\mu(x))\right)\right)= \\
& =\widehat{S}_{a, b}^{(k+1) / 2}\left(\frac{\mu(x)^{t}}{\beta \lambda_{1}^{2}}\right)=\widehat{S}_{a, b}^{(k+1) / 2}\left(c \delta(x)^{t}\right),
\end{aligned}
$$

where $c=1 /\left(\beta \lambda_{1}^{2}\right), \delta(x)=\mu(x)$ and $t>2$ is odd. This is case (III) in Theorem 1.

Next suppose that, in (18), the standard pair $\left(f_{1}(x), g_{1}(x)\right)$ is of the second kind. If $\left(f_{1}(x), g_{1}(x)\right)=\left(x^{2},\left(\alpha x^{2}+\beta\right) v(x)^{2}\right)$, then a calculation from (24) leads to $\ell(x)=x / \lambda_{1}^{2}$, and from (18) we obtain

$$
\begin{aligned}
& g(x)=\widehat{S}_{a, b}^{(k+1) / 2}\left(\ell\left(g_{1}(\mu(x))\right)\right)= \\
= & \widehat{S}_{a, b}^{(k+1) / 2}\left(\frac{\left(\alpha x^{2}+\beta\right) v(\mu(x))^{2}}{\lambda_{1}^{2}}\right)=\widehat{S}_{a, b}^{(k+1) / 2}\left(\left(\alpha \delta(x)^{2}+\beta\right) q(x)^{2}\right),
\end{aligned}
$$


where $\delta(x)=\mu(x)$ and $q(x)=v(\mu(x)) / \lambda_{1}$. So we are in case (IV) of Theorem 1 .

If $\left(f_{1}(x), g_{1}(x)\right)=\left(\left(\alpha x^{2}+\beta\right) v(x)^{2}, x^{2}\right)$, then since $\operatorname{deg} f_{1}(x)=2$, $v(x)$ is a constant polynomial and we have

$$
\begin{aligned}
g(x)= & \widehat{S}_{a, b}^{(k+1) / 2}\left(\ell\left(g_{1}(\mu(x))\right)\right)= \\
& =\widehat{S}_{a, b}^{(k+1) / 2}\left(\ell_{1} \mu(x)^{2}+\ell_{0}\right)=\widehat{S}_{a, b}^{(k+1) / 2}\left(\left(\ell_{1} \delta(x)^{2}+\ell_{0}\right) q(x)^{2}\right),
\end{aligned}
$$

with $\delta(x)=\mu(x)$ and $q(x) \equiv 1$. Again, we arrived at case (IV) of Theorem 1 .

Now, if the standard pair $\left(f_{1}(x), g_{1}(x)\right)$ is of the third kind, then $\left(f_{1}(x), g_{1}(x)\right)=\left(D_{2}\left(x, \alpha^{t}\right), D_{t}\left(x, \alpha^{2}\right)\right)$ with $t$ being odd. Let us substitute $f_{1}(x)=x^{2}-2 \alpha^{t}$ into $(24)$ to deduce that $\ell(x)=\left(x+2 \alpha^{t}\right) / \lambda_{1}^{2}$, whence

$$
g(x)=\widehat{S}_{a, b}^{(k+1) / 2}\left(\ell\left(g_{1}(\mu(x))\right)\right)=\widehat{S}_{a, b}^{(k+1) / 2}\left(\frac{D_{t}\left(\mu(x), \alpha^{2}\right)+2 \alpha^{t}}{\lambda_{1}^{2}}\right) .
$$

It follows from Proposition 7 that $-2 \alpha^{t} / \lambda_{1}^{2}$ is an extremum of the polynomial $D_{t}\left(\mu(x), \alpha^{2}\right) / \lambda_{1}^{2}$, which is of type $(1,2, \ldots, 2)$ as $t$ is odd. This implies that $\left(D_{t}\left(\mu(x), \alpha^{2}\right)+2 \alpha^{t}\right) / \lambda_{1}^{2}=\delta(x) q(x)^{2}$ for some $\delta(x), q(x) \in$ $\mathbb{Q}[x]$ with $\operatorname{deg} \delta(x)=1$. Hence $g(x)$ is of the form as in Theorem 1 (II).

Finally, consider the case when $\left(f_{1}(x), g_{1}(x)\right)$ is a standard pair of the fourth kind. Then

$$
\left(f_{1}(x), g_{1}(x)\right)=\left(\frac{D_{2}(x, \alpha)}{\alpha}, \frac{D_{t}(x, \beta)}{\beta^{(t / 2)}}\right)
$$

where $t$ is even. Substituting this into (24), it is easy to calculate that $\ell(x)=(\alpha x+2 \alpha) / \lambda_{1}^{2}$. Hence, by (18), we obtain

$$
\begin{aligned}
g(x)=\widehat{S}_{a, b}^{(k+1) / 2}\left(\ell\left(g_{1}(\mu(x))\right)\right)= & \\
= & \widehat{S}_{a, b}^{(k+1) / 2}\left(\frac{\alpha \beta^{-t / 2} D_{t}(\mu(x), \beta)+2 \alpha}{\lambda_{1}^{2}}\right) .
\end{aligned}
$$

Now Proposition 7 implies that

$$
-\frac{2 \beta^{t / 2} \alpha \beta^{-t / 2}}{\lambda_{1}^{2}}=-\frac{2 \alpha}{\lambda_{1}^{2}}
$$

is one of the two extrema of the polynomial $\alpha \beta^{-t / 2} D_{t}(\mu(x), \beta) /\left(\lambda_{1}^{2}\right)$ and it is of type $(2,2, \ldots, 2)$ as $t$ is even. It follows that

$$
\frac{\alpha \beta^{-t / 2} D_{t}(\mu(x), \beta)+2 \alpha}{\lambda_{1}^{2}}=q(x)^{2}
$$


for some $q(x) \in \mathbb{Q}[x]$. Thus $g(x)$ is of type $(\mathrm{V})$ in Theorem 1 . This completes the proof.

\section{Proofs of Theorems 2 And 3}

We discuss the proofs of Theorems 2 and 3 jointly by introducing the following notation. Let $\mathrm{T}_{a, b}^{k \pm}(x) \in\left\{\mathrm{T}_{a, b}^{k+}(x), \mathrm{T}_{a, b}^{k-}(x)\right\}$ and similarly, let $\widehat{T}_{a, b}^{m \pm}(x) \in\left\{\widehat{T}_{a, b}^{m+}(x), \widehat{T}_{a, b}^{m-}(x)\right\}$. Now equations (14) and (15) can be written in the common form

$$
\mathrm{T}_{a, b}^{k \pm}(x)=g(y),
$$

where $g(y)$ is a rational polynomial of degree at least 3 .

Before starting the proof we need the following auxiliary results besides the ones from the previous sections.

The first one is a deep result of Rakaczki [21] concerning the root structure of shifted Euler polynomials.

Proposition 8. Let $m \geq 7$ be an integer. Then the shifted Euler polynomial $E_{m}(x)+b$ has at least three simple zeros for arbitrary complex number $b$.

The following result is Lemma 11 in $[22]$. Let $c_{1}, e_{1} \in \mathbb{Q} \backslash\{0\}$ and $c_{0}, e_{0} \in \mathbb{Q}$.

Lemma 9. The polynomial $E_{k}\left(c_{1} x+c_{0}\right)$ is neither of the form $e_{1} x^{q}+e_{0}$ with $q \geq 3$, nor of the form $e_{1} D_{\nu}(x, \delta)+e_{0}$, where $D_{\nu}(x, \delta)$ is the $\nu$-th Dickson polynomial with $\nu>4, \delta \in \mathbb{Q} \backslash\{0\}$.

The next lemma is a simple consequence of the previous one. Further, it is an analogue of Lemmas 5 and 6 from the preceding section.

Lemma 10. None of the polynomials $T_{a, b}^{k+}\left(c_{1} x+c_{0}\right)$ and $T_{a, b}^{k-}\left(c_{1} x+c_{0}\right)$ are either of the form $e_{1} x^{q}+e_{0}$ with $q \geq 3$, or of the form $e_{1} D_{\nu}(x, \delta)+e_{0}$, where $D_{\nu}(x, \delta)$ is the $\nu$-th Dickson polynomial with $\nu>4, \delta \in \mathbb{Q} \backslash\{0\}$.

Proof. We detail the proof only for the 'positive' case. For the 'negative' case the argument is essentially the same.

Since $\mathrm{T}_{a, b}^{k+}\left(c_{1} x+c_{0}\right)=a^{k} / 2\left(E_{k}(b / a)+E_{k}\left(c_{1} x+c_{0}+b / a\right)\right)$, we have

$$
E_{k}\left(c_{1} x+c_{0}+\frac{b}{a}\right)=\frac{2}{a^{k}} \mathrm{~T}_{a, b}^{k+}\left(c_{1} x+c_{0}\right)-E_{k}\left(\frac{b}{a}\right) .
$$

Put $c_{0}^{\prime}=c_{0}+b / a, e_{1}^{\prime}=\left(2 e_{1}\right) /\left(a^{k}\right)$ and $e_{0}^{\prime}=\left(2 e_{0}\right) /\left(a^{k}\right)-E_{k}(b / a)$.

Now if $\mathrm{T}_{a, b}^{k+}\left(c_{1} x+c_{0}\right)=e_{1} x^{q}+e_{0}$ for some $q \geq 3$, then we obtain

$$
E_{k}\left(c_{1} x+c_{0}^{\prime}\right)=e_{1}^{\prime} x^{q}+e_{0}^{\prime} .
$$


This contradicts Lemma 9.

Similarly, if $\mathrm{T}_{a, b}^{k+}\left(c_{1} x+c_{0}\right)=e_{1} D_{\nu}(x, \delta)+e_{0}$ for some $\nu>4$ and $\delta \in \mathbb{Q} \backslash\{0\}$, then by (33) we have

$$
E_{k}\left(c_{1} x+c_{0}^{\prime}\right)=e_{1}^{\prime} D_{\nu}(x, \delta)+e_{0}^{\prime},
$$

contradicting again Lemma 9.

Proof of Theorems 2 and 3. Suppose that equation (32) has infinitely many solutions in integers $x, y$. Then by Proposition 3 , there exist $\varphi(x) \in \mathbb{Q}[x]$ and linear polynomials $\lambda(x), \mu(x) \in \mathbb{Q}[x]$ such that

$$
\mathrm{T}_{a, b}^{k \pm}(x)=\varphi\left(f_{1}(\lambda(x))\right) \quad \text { and } \quad g(x)=\varphi\left(g_{1}(\mu(x))\right),
$$

where $\left(f_{1}(x), g_{1}(x)\right)$ is a standard pair over $\mathbb{Q}$. From $\operatorname{deg} \mathrm{T}_{a, b}^{k \pm}(x)=k$ and from Proposition 2 we infer that

$$
\operatorname{deg} \varphi(x) \in\left\{1, \frac{k}{2}, k\right\} .
$$

Suppose first that $\operatorname{deg} \varphi(x)=k$. Then (34) implies that $\operatorname{deg} f_{1}(x)=$ 1. Therefore $\mathrm{T}_{a, b}^{k \pm}(x)=\varphi(t(x))$, for a linear polynomial $t(x) \in \mathbb{Q}[x]$. Clearly, $t^{-1}(x) \in \mathbb{Q}[x]$ is also linear. Thus, by (34), we get that $\mathrm{T}_{a, b}^{k \pm}\left(t^{-1}(x)\right)=\varphi\left(t\left(t^{-1}(x)\right)\right)=\varphi(x)$, whence

$$
g(x)=\varphi\left(g_{1}(\mu(x))\right)=\mathrm{T}_{a, b}^{k \pm}\left(t^{-1}\left(g_{1}(\mu(x))\right)\right)=\mathrm{T}_{a, b}^{k \pm}(q(x)),
$$

where $q(x)=t^{-1}\left(g_{1}(\mu(x))\right)$. So, if equation (32) has infinitely many solutions, then $g(x)$ is of the form (I) in Theorem 2 or 3 , respectively.

Next we assume that $\operatorname{deg} \varphi(x)=1$. Then there exist $\varphi_{1}, \varphi_{0} \in \mathbb{Q}$ with $\varphi_{1} \neq 0$ such that $\varphi(x)=\varphi_{1} x+\varphi_{0}$. We study now the five kinds of standard pairs over $\mathbb{Q}$. In view of $k \geq 7$ and $\operatorname{deg} g(x) \geq 3$, we see that, in (34), the standard pair $\left(f_{1}(x), g_{1}(x)\right)$ cannot be of the second or the fifth kind.

Now in (34), let $\left(f_{1}(x), g_{1}(x)\right)$ assumed to be a standard pair of the first kind. Then we have either

(i) $\mathrm{T}_{a, b}^{k \pm}\left(\lambda^{-1}(x)\right)=\varphi_{1} x^{t}+\varphi_{0}$, or

(ii) $\mathrm{T}_{a, b}^{k \pm}\left(\lambda^{-1}(x)\right)=\varphi_{1} \alpha x^{r} q(x)^{t}+\varphi_{0}$, where $0 \leq r<t,(r, t)=1$ and $r+\operatorname{deg} q(x)>0$.

In the first case (i), we obtain a contradiction by Lemma 10 since $t=k \geq 7$.

In the case (ii), since

$$
\mathrm{T}_{a, b}^{k \pm}\left(\lambda^{-1}(x)\right)-\varphi_{0}=\frac{a^{k}}{2}\left(E_{k}\left(\lambda^{-1}(x)+\frac{b}{a}\right) \pm E_{k}\left(\frac{b}{a}\right)-\frac{2 \varphi_{0}}{a^{k}}\right),
$$


and since the root structure of a polynomial remains the same if the variable of the polynomial is replaced by a linear polynomial of it, we infer by Proposition 8 that $\mathrm{T}_{a, b}^{k \pm}\left(\lambda^{-1}(x)\right)-\varphi_{0}$ has at least three simple zeros. By the assumptions on $r$ and $t$, this implies that $r=0$ and $t=\operatorname{deg} g(x)=1$, which contradicts $\operatorname{deg} g(x) \geq 3$.

Finally, suppose that $\left(f_{1}(x), g_{1}(x)\right)$ is a standard pair of the third or the fourth kind over $\mathbb{Q}$. Then we obtain

$$
\mathrm{T}_{a, b}^{k \pm}\left(\lambda^{-1}(x)\right)=\varphi_{1}^{\prime} D_{k}(x, \delta)+\varphi_{0},
$$

where $\varphi_{1}^{\prime} \in\left\{\varphi_{1}, a^{k / 2} \varphi_{1}\right\}$ and $\delta \in \mathbb{Q} \backslash\{0\}$. This is a contradiction by Lemma 10 since $k \geq 7$.

The case $\operatorname{deg} \varphi(x)=k / 2$. Clearly, $k$ is then even, and from (34) we observe that $\operatorname{deg} f_{1}(x)=2$. Hence it follows that, in $(34),\left(f_{1}(x), g_{1}(x)\right)$ cannot be a standart pair of the fifth kind. Further, we obtain a nontrivial decomposition of $\mathrm{T}_{a, b}^{k \pm}(x)$, which by Proposition 2 implies that there exists a linear polynomial $\ell(x)=\ell_{1} x+\ell_{0}$ over $\mathbb{Q}$ such that

$$
\varphi(x)=\widehat{T}_{a, b}^{k / 2 \pm}(\ell(x)) \quad \text { and } \quad \ell\left(f_{1}(\lambda(x))\right)=\left(x+\frac{b}{a}-\frac{1}{2}\right)^{2} .
$$

Again, we study the remaining kinds of standard pairs.

First, we consider the case when, in $(34),\left(f_{1}(x), g_{1}(x)\right)$ is a standard pair of the first kind. If $f_{1}(x)=x^{t}$, then by $\operatorname{deg} f_{1}(x)=2$, we have $\left(f_{1}(x), g_{1}(x)\right)=\left(x^{2}, \alpha x p(x)^{2}\right)$. Putting $\lambda(x)=\lambda_{1} x+\lambda_{0}$, (36) takes the form $\ell\left(\left(\lambda_{1} x+\lambda_{0}\right)^{2}\right)=(x+b / a-1 / 2)^{2}$, whence an easy calculation gives $\ell(x)=x / \lambda_{1}^{2}$. Substituting this to (34), we obtain

$$
g(x)=\widehat{T}_{a, b}^{k / 2 \pm}\left(\ell\left(g_{1}(\mu(x))\right)\right)=\widehat{T}_{a, b}^{k / 2 \pm}\left(\frac{\alpha \mu(x) p(\mu(x))^{2}}{\lambda_{1}^{2}}\right)
$$

So $g(x)$ is of the form (III) with $\delta(x)=\alpha \mu(x) / \lambda_{1}^{2}$ and $q(x)=p(\mu(x))$.

In the switched case $\left(f_{1}(x), g_{1}(x)\right)=\left(\alpha x^{r} p(x)^{t}, x^{t}\right)$, where $r<t$, $(r, t)=1$ and $r+\operatorname{deg} p(x)>0, \operatorname{deg} f_{1}(x)=2$ implies that one of the following cases occurs:

(A) $r=0, t=1$ and $\operatorname{deg} p(x)=2$, or

(B) $r=2, t>2$ is odd and $p(x)$ is a constant polynomial.

In case $(\mathrm{A})$ we have $g_{1}(x)=x$, whence from (34) and (36) we obtain

$$
\begin{aligned}
g(x)=\widehat{T}_{a, b}^{k / 2 \pm}\left(\ell\left(g_{1}(\mu(x))\right)\right) & = \\
& =\widehat{T}_{a, b}^{k / 2 \pm}(\ell(\mu(x)))=\widehat{T}_{a, b}^{k / 2 \pm}\left(\delta(x) q(x)^{2}\right),
\end{aligned}
$$

where $\delta(x)=\ell(\mu(x))$ and $q(x) \equiv 1$. Thus $g(x)$ is again of type (III). 
In the second case (B), we can write $f_{1}(x)=\beta x^{2}$, with $\beta=\alpha p(x)^{t} \in$ $\mathbb{Q} \backslash\{0\}$. Substituting this to (36), we deduce that $\ell(x)=x /\left(\beta \lambda_{1}^{2}\right)$, whence, by (34), we get

$$
\begin{aligned}
& g(x)=\widehat{T}_{a, b}^{k / 2 \pm}\left(\ell\left(g_{1}(\mu(x))\right)\right)= \\
&=\widehat{T}_{a, b}^{k / 2 \pm}\left(\frac{\mu(x)^{t}}{\beta \lambda_{1}^{2}}\right)=\widehat{T}_{a, b}^{k / 2 \pm}\left(c \delta(x)^{t}\right),
\end{aligned}
$$

where $c=1 /\left(\beta \lambda_{1}^{2}\right), \delta(x)=\mu(x)$ and $t>2$ is odd. This is case (IV) in Theorems 2 or 3 , respectively.

Next let, in (34), be a the standard pair $\left(f_{1}(x), g_{1}(x)\right)$ of the second kind. If $\left(f_{1}(x), g_{1}(x)\right)=\left(x^{2},\left(\alpha x^{2}+\beta\right) v(x)^{2}\right)$, then a calculation from (36) yields $\ell(x)=x / \lambda_{1}^{2}$, and by (34) we have

$$
\begin{aligned}
g(x) & =\widehat{T}_{a, b}^{k / 2 \pm}\left(\ell\left(g_{1}(\mu(x))\right)\right)= \\
= & \widehat{T}_{a, b}^{k / 2 \pm}\left(\frac{\left(\alpha x^{2}+\beta\right) v(\mu(x))^{2}}{\lambda_{1}^{2}}\right)=\widehat{T}_{a, b}^{k / 2 \pm}\left(\left(\alpha \delta(x)^{2}+\beta\right) q(x)^{2}\right),
\end{aligned}
$$

where $\delta(x)=\mu(x)$ and $q(x)=v(\mu(x)) / \lambda_{1}$. So we are in case (V) of our Theorems.

In the switched case $\left(f_{1}(x), g_{1}(x)\right)=\left(\left(\alpha x^{2}+\beta\right) v(x)^{2}, x^{2}\right)$, since $\operatorname{deg} f_{1}(x)=2, v(x)$ is a constant polynomial and

$$
g(x)=\widehat{T}_{a, b}^{k / 2 \pm}\left(\ell\left(g_{1}(\mu(x))\right)\right)=\quad=\widehat{T}_{a, b}^{k / 2 \pm}\left(\left(\ell_{1} \mu(x)^{2}+\ell_{0}\right) q(x)^{2}\right),
$$

where $q(x) \equiv 1$. Thus, we arrived again at case $(\mathrm{V})$ with $\delta(x)=\mu(x)$ and $\alpha=\ell_{1}, \beta=\ell_{0}$.

Now, if the standard pair $\left(f_{1}(x), g_{1}(x)\right)$ is of the third kind over $\mathbb{Q}$, then $\left(f_{1}(x), g_{1}(x)\right)=\left(D_{2}\left(x, \alpha^{t}\right), D_{t}\left(x, \alpha^{2}\right)\right)$ with $t$ being odd. Let us substitute $f_{1}(x)=x^{2}-2 \alpha^{t}$ into (36) to deduce that $\ell(x)=\left(x+2 \alpha^{t}\right) / \lambda_{1}^{2}$, whence

$$
g(x)=\widehat{T}_{a, b}^{k / 2 \pm}\left(\ell\left(g_{1}(\mu(x))\right)\right)=\widehat{T}_{a, b}^{k / 2 \pm}\left(\frac{D_{t}\left(\mu(x), \alpha^{2}\right)+2 \alpha^{t}}{\lambda_{1}^{2}}\right) .
$$

It follows from Proposition 7 that $-2 \alpha^{t} / \lambda_{1}^{2}$ is an extremum of the polynomial $D_{t}\left(\mu(x), \alpha^{2}\right) / \lambda_{1}^{2}$, which is of type $(1,2, \ldots, 2)$ as $t$ is odd. Hence $\left(D_{t}\left(\mu(x), \alpha^{2}\right)+2 \alpha^{t}\right) / \lambda_{1}^{2}=\delta(x) q(x)^{2}$ for some $\delta(x), q(x) \in \mathbb{Q}[x]$ with $\operatorname{deg} \delta(x)=1$. We deduce, that $g(x)$ is of type (III).

Finally, consider the case when $\left(f_{1}(x), g_{1}(x)\right)$ is a standard pair of the fourth kind over $\mathbb{Q}$. Then

$$
\left(f_{1}(x), g_{1}(x)\right)=\left(\frac{D_{2}(x, \alpha)}{\alpha}, \frac{D_{t}(x, \beta)}{\beta^{(t / 2)}}\right),
$$


with an even $t$. Substituting this into (36), an easy calculation yields $\ell(x)=(\alpha x+2 \alpha) / \lambda_{1}^{2}$, whence, by (34), we obtain

$$
\begin{aligned}
g(x)=\widehat{T}_{a, b}^{k / 2 \pm}\left(\ell\left(g_{1}(\mu(x))\right)\right) & = \\
& =\widehat{T}_{a, b}^{k / 2 \pm}\left(\frac{\alpha \beta^{-t / 2} D_{t}(\mu(x), \beta)+2 \alpha}{\lambda_{1}^{2}}\right) .
\end{aligned}
$$

Now from Proposition 7 we infer that

$$
-\frac{2 \beta^{t / 2} \alpha \beta^{-t / 2}}{\lambda_{1}^{2}}=-\frac{2 \alpha}{\lambda_{1}^{2}}
$$

is one of the two extrema of the polynomial $\alpha \beta^{-t / 2} D_{t}(\mu(x), \beta) /\left(\lambda_{1}^{2}\right)$ and it is of type $(2,2, \ldots, 2)$ as $t$ is even. Therefore we have

$$
\frac{\alpha \beta^{-t / 2} D_{t}(\mu(x), \beta)+2 \alpha}{\lambda_{1}^{2}}=q(x)^{2}
$$

for some $q(x) \in \mathbb{Q}[x]$. Thus $g(x)$ is of type (II), and the proof is complete.

\section{ACKNowledGements}

Research was supported by the Hungarian Academy of Sciences and by the OTKA grant NK104208.

\section{REFERENCES}

1. A. BAzsó, On alternating power sums of arithmetic progressions, Integral Transforms Spec. Funct., 24 (2013), 945-949.

2. A. Bazsó, D. Kreso, F. LucA and Á. Pintér, On equal values of power sums of arithmetic progressions, Glas. Mat. Ser. III, 47 (2012), 253-263.

3. A. Bazsó, Á. Pintér and H. M. Srivastava, On a refinement of Faulhaber's Theorem concerning sums of powers of natural numbers, Appl. Math. Letters, 25 (2012), 486-489.

4. M. A. Bennett, A superelliptic equation involving alternating sums of powers, Publ. Math. Debrecen, 79 (2011), 317-324.

5. M. A. Bennett, K. Győry and Á. Pintér, On the Diophantine equation $1^{k}+2^{k}+\cdots+x^{k}=y^{n}$, Compos. Math., 140 (2004), 1417-1431.

6. Y. F. BILU, Quadratic factors of $f(x)-g(y)$, Acta Arith., 90 (1999), 341-355.

7. Y. F. Bilu and R. F. Tichy, The Diophantine equation $f(x)=g(y)$, Acta Arith., 95 (2000), 261-288.

8. Y. F. Bilu, B. Brindza, P. Kirschenhofer, Á. Pintér and R. F. Tichy, Diophantine equations and Bernoulli polynomials (with an Appendix by A. Schinzel), Compositio Math., 131 (2002), 173-188.

9. J. Brillhart, On the Euler and Bernoulli polynomials, J. Reine Angew. Math., 234 (1969), 45-64.

10. B. BRINDZA, On some generalizations of the diophantine equation $1^{k}+2^{k}+$ $\ldots+x^{k}=y^{z}$, Acta Arith., 44 (1984), 99-107. 
11. K. Dilcher, On a Diophantine equation involving quadratic characters, Compositio Math. 57 (1986), 383-403.

12. K. GYöRY and Á. PInTÉR, On the equation $1^{k}+2^{k}+\ldots+x^{k}=y^{n}$, Publ. Math. Debrecen, 62 (2003), 403-414.

13. K. Győry, R. Tijdeman and M. Voorhoeve, On the equation $1^{k}+2^{k}+$ $\ldots+x^{k}=y^{z}$, Acta Arith., 37 (1980), 234-240.

14. F. T. Howard, Sums of powers of integers via generating functions, Fibonacci Quart., 34 (1996), 244-256.

15. M. Jacobson, Á. Pintér and P. G. Walsh, A computational approach for solving $y^{2}=1^{k}+2^{k}+\cdots+x^{k}$, Math. Comp., 72 (2003), 2099-2110.

16. É. LuCAs, Problem 1180, Nouvelles Ann. Math., 14 (1875), 336.

17. M. KUlKarni and B. SURY, Diophantine equations with Bernoulli polynomials, Acta Arith., 116 (2005), 25-34.

18. M. Kulkarni and B. Sury, A class of Diophantine equations involving Bernoulli polynomials, Indag. Math. (N.S.), 16 (2005), 51-65.

19. M. KulKarni and B. Sury, On the Diophantine equation $1+x+\frac{x^{2}}{2 !}+\ldots+$ $\frac{x^{n}}{n !}=g(y)$., In: Diophantine equations, 121-134, Tata Inst. Fund. Res. Stud. Math., Mumbai, 2008.

20. Cs. Rakaczki, On the Diophantine equation $S_{m}(x)=g(y)$, Publ. Math. Debrecen, 65 (2004), 439-460.

21. Cs. Rakaczki, On the simple zeros of shifted Euler polynomials, Publ. Math. Debrecen, 79 (2011), 623-636.

22. Cs. RAKaczKI and D. Kreso, Diophantine equations with Euler polynomials, Acta Arith., 161 (2013), 267-281.

23. J. J. SChäFFER The equation $1^{p}+2^{p}+3^{p}+\cdots+n^{p}=m^{q}$ Acta Math., 95 (1956), 155-189.

24. A. Schinzel, Polynomials with special regard to reducibility, Cambridge University Press, 2000.

25. J. Urbanowicz, On the equation $f(1) 1^{k}+f(2) 2^{k}+\ldots+f(x) x^{k}+R(x)=b y^{z}$, Acta Arith., 51 (1988), 349-368.

26. J. Urbanowicz, On diophantine equations involving sums of powers with quadratic characters as coefficients, I., Compositio Math., 92 (1994), 249-271.

27. J. Urbanowicz, On diophantine equations involving sums of powers with quadratic characters as coefficients, II., Compositio Math., 102 (1996), 125140.

28. M. Voorhoeve, K. Győry and R. Tijdeman, On the diophantine equation $1^{k}+2^{k}+\ldots+x^{k}+R(x)=y^{z}$, Acta Math., 143 (1979), 1-8; Corr. 159 (1987), $151-152$.

ANDRÁS BAZSÓ

InSTITUTE OF MATHEMATICS

MTA-DE Research Group "Equations Functions and Curves"

Hungarian Academy of Sciences and University of Debrecen

P.O. Box 12, H-4010 Debrecen, Hungary

E-mail address: bazsoa@science.unideb.hu 